\title{
IMAGE PATTERN REOOGNITION BY QUAD STRING ARRAY APPROACH
}

Yung-Lung Ma, Chialo Ma and Tsing-Yee Tu

Institute of Electrical Engineering

National Taiwan University, Taipei, Taiwan, R.O.C.

Abstract

How to use one-chip processor and small size memory to recognize the shape of object is the most important topic in modern industrial application. Usually, it is impossible to get the front view of fast flying object , the image taken at the view direction are called plane projection images The plane projection image is composed of "object" and "background", that is what we say pixel recurgion image. The image processing by the using of pixel recursion is the most simple and fast approach. The pixel recursion image can be achieved by the scanning, and the image after size and orientation normalization can be recognized by the Quad String Array (SSA) approach.

The size normalization can be achieved by the scanning device and the orientation normalization can be handled by the Circular Layer string method. In the orientation normalization, the principle axis of unknow image is achieved by rotation and compared with the standard patterns which are pre-estimation. The uniknown image can be divided into serval subimage and each subimage is coded by the Vertical String Array. By the using of Vertical string Array, the shape of unknown object can be described precisely. In this paper, the experiment results of Vertical String Array. Random Walk and Gauss-Markov-R-process are also discussed.

\section{INTRODUCTION}

The statistical approach was commonly used in the $1960^{\prime} \mathrm{s}$ to process the 2-D or 3-D image patterns. The recognition was achieved by storing the sample data of a pattern in the memory which were then compared with the random input pattern. A Markov approach $[1,2,3]$ was adopted to recognize the 2-D pattern into 10 layers structured as a pyranid. The variation of each mesh was represented by a transfer function. [4] describes the circular layer method to implement the orientation ,[5] [6] used the complexity to recognize the inage.

\section{KOLMOGOROV OOMPLEXITY}

The digital picture point method is a general approach for image recognition. Each pixel is expressed by the brightness level ranged from 0 to 256. Assumption 2-1:

The length, the area, even the size of any pattern in arbitrary direction are all finite. Therefore, the string array of pixels can be used for recognizing any feature of a 2-D image picture.

Definition 2-1:

Let $(0,1)^{*}$ express an optinal binary string of finite length. The first $n$ terms of input pattern sequence are denoted as $X(n)$, where

$x(n)=\left(x_{1}, x_{2}, \ldots, x_{n}\right)$

Let $A$ be an automaton which can transfer the random input into an optimally logical output string array, such that

$A:\{0,1\}^{*} x$ N $\rightarrow(0,1)^{*}$,

where $\mathrm{N}$ is the number of extracted features. Definition 2-2: Complexity production
A binary data representation has the complexity if it can be expressed as follows

$\left|x \in\{0,1\}^{*}: K(X)=k\right|<2^{k}$.

where $K(X)$ is called Kolmogorov complexity program.

Rule 2-2: The production of Kolmogorov complexity program

Let $\ell(X)$ be the length of the sequence $X(m)$ and $X(m)$ $/ n$ be a probability of the occurrence of pixeled in a string of length $n$. $X(m)$ represents the total number of appearances of pixels.

$K(X)$ can be expressed by $\ell(X)$; as follows

$\mathrm{K}\left[\mathrm{X}(\mathrm{m} / \mathrm{n})=\min _{\mathrm{A}(\mathrm{p}, \mathrm{n})=\mathrm{X}(\mathrm{m})} \ell(\mathrm{p})\right.$,

where $m=1,2, \ldots, n$ and $p$ is the probability of successful appearance of the image pixels. $A(p, n)$ means that the automaton can convert the successful event among total number of features $n$ with probability $p$ into. $l$ (p) represents the length of the string where object pixels occurred.

\section{STRING QUAD ARRAY ANALYSIS}

Define: $s \in S, s=1,2, \ldots, s$.

where $s$ is the number of string components.

Definition 4-1:

The object in "pixel recursion" is defined as " 1 " and the background in "pixel recursion" is defined as " 0 ". Definition 3-2:

The area of pseudo Quad plane in scanning process is similar to discrimination function, in this paper, is defined as "Recognition Potential function" or "QuadArray function".

Definition 3-3:

The pixel number of horizontal scanning line is $m$ and the pixel number of vertical scanning line is $n$, where $m=1,2, \ldots i, \ldots M, 1<M<\infty ; n=1,2, \ldots j, \ldots N, 1<N<\infty$. Definition 3-4:

the number of vertical pixel $n$ and horizontal pixel m are decided by the real edge of object image, such that the Quad plane potential function $Q_{s}(m, n)$ is a function of $(m, n)$.

(1) Object image adge is monodescending sense in right hand side of topmost point.

Definition 3-5:

Object image edge is monodescending scense as show in Fig.3. The raster line on the right side of topmost point is divided into $S$ stages, $Q_{s}$ is the area belween $S$-th stage raster and corresponding object inage edge $w_{s}$. The potential function sequence is defined as $\tilde{Q}$,

$\tilde{Q}_{i}=\left[Q_{1}, Q_{2}, \ldots Q_{s}, \ldots Q_{S}\right]$.

This potential function as called as Quad String Array Corollary 3-1:

As shown in Fig. 3 , if object edge is monodecreasing, no matter it is linear or not, we can divide the raster line in equal division $m$. But the vertical pixel number of $Q$ is decided by the shape of object edge, and the length sequence is $N$, 
$\mathrm{N}=\left(\mathrm{N}_{1}, \mathrm{~N}_{\dot{2}}, \ldots \mathrm{N}_{\mathrm{g}}, \ldots \mathrm{N}_{\mathrm{S}}\right)$

Then

$Q_{1}=R_{1}(0,0)+T_{1}\left(m, n_{1}\right) ;$

$Q_{2}=R_{2}\left(m, n_{1}\right)+T_{2}\left(m, n_{2}-n_{1}\right)$

$\dot{2}$

$Q_{s}=\dot{R}_{s}\left(m, n_{s-1}\right)+T_{2}\left(m_{1} n_{2}-n_{1}\right)$

where $R($.$) is the rectangular quad; T($.$) is the$ triangle quad and $R(0,0)=0, T(0,0)=0$.

(2) Object image edge as fluctuating wave form. Corollary 3-2:

-As show in Fig, 4, the fluctuating wave form is another form of monodescending and monoincreasing wave form.

Definition 3 6:

The Quad String Array shown in Fig. 4 is defined as Q,

$\tilde{Q}_{I I}=\left\{Q_{1}, Q_{2}, \ldots Q_{s}, \ldots Q_{S}\right\}$

The horizontal interval of $Q_{s}$ is decided by the interval between the valley point $v_{s}$ and the neighbor top point $\mathrm{P}_{\mathbf{8 - 1}}$. The intervals between each quads may not be equal.

Corollary 3-3:

each quad of fluctuant edge can be divided into equal M's, divisions horizontally. The quad $Q_{1 \mathrm{~m}}$ is in

monodecreasing sense for odd 1 , and is in monoincreasing sense for 1 .Then

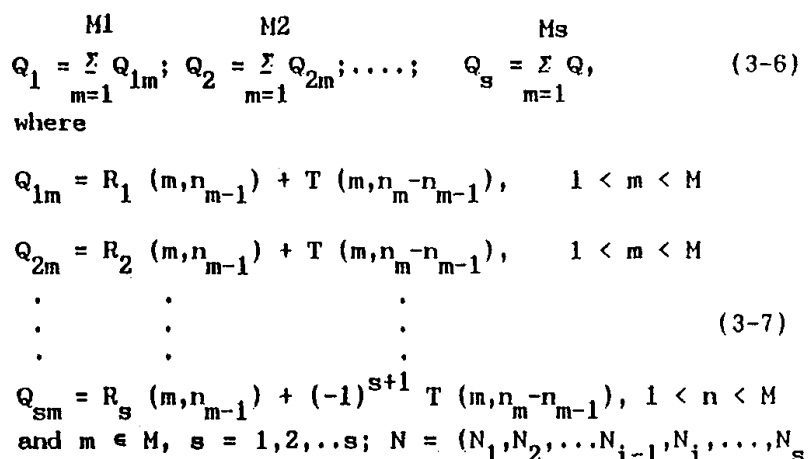
,$N_{S}$ ). The sign of triangular quad is plus for the edge in monodecreasing scene and minus for the edge in monocreasing scene.

IV. REOOGNITION ALOORIIIII

Assumption 4-1:

If the object inage edges are divided into small partitions then each interval will be linear no matter how shape the original edges are.

Assumption 1-2:

If the object image edges are an irregular fluctuant wave form, then the quad plane domain will be connvexity. The quad domain will be convex if edge fluctuates downward and be concave if edge fluctuates upward.

Assumption 6-3:

The string QUAD Sequence Array $\tilde{Q}_{I I}$
$\tilde{Q}_{I I}=\left(Q_{1}, Q_{2}, \ldots Q_{s}, \ldots Q_{S}\right)$

$Q_{s}$ is the s-th stage Quad Potential Function.

$n=\left(w_{1}, w_{2}, \ldots w_{s}, \ldots w_{s}\right)$

Definition 4-1:

The sample space of Quad Potential Function.sequence is $Q$ sample, then

$\tilde{Q}^{\text {samp }}=\left(Q_{1}{ }^{\text {samp }}, Q_{2}{ }^{\text {samp }}, \ldots Q_{S}{ }^{\text {samp }}, \ldots Q_{S}{ }^{\text {samp })}\right.$

Lerma 4-1:

The Quad Potential Function Sequence of new input flying object image is $Q$, then

$\tilde{Q}=\left(Q_{1}, Q_{2}, \ldots Q_{s}, \ldots Q_{S}\right)$

The error sequence between unknown object and standard pattern is $e$, then e can be defined as following,

$$
\text { where } \begin{aligned}
\tilde{e} & =\left(e_{1}, e_{2}, \ldots e_{s}, \ldots e_{s}\right) \\
\text { or } \quad e_{1} & =Q_{1}-Q_{1} \text { samp } \\
e_{1} & =\left|Q_{1}-Q_{1}{ }^{\text {samp }}\right|
\end{aligned}
$$

The Risk Function of Quad Potential Function Sequence is:

$$
E=\left(\left|e_{1}\right|,\left|e_{2}\right|, \ldots\left|e_{s}\right|, \ldots\left|e_{s}\right|\right)=\underset{s=1}{\#}\left|e_{s}\right| \text {, }
$$

where "\#" is defined as the String Connection Function - The minimum Risk Function (or Performance index) be defined as $\mathrm{J}$,

$J=\operatorname{mini}_{s} E=\operatorname{mini}_{s}\left(\left|e_{i}\right|,\left|e_{2}\right| \ldots\left|e_{s}\right|, \ldots\left|e_{s}\right|\right)$

The object edge $w_{s}$ is one side of the right-triangular potential function $\mathrm{T}_{s}\left(\mathrm{~m}, \mathrm{n}_{\mathrm{s}}-\mathrm{n}_{\mathrm{s}-1}\right)$ and $\mathrm{W}_{\mathrm{s}}$ may be nonlinear.

If the unknown object image is similar or resemble to the standard pattern then it must satisfied the following optimum threshold condition.

$\mathrm{e}_{\mathrm{s}}<\mathrm{Th}^{*}(\mathrm{~s})=\mathrm{T}_{\mathrm{s}}{ }^{\text {samp }}\left(\mathrm{m}, \mathrm{n}_{\mathrm{s}}{ }^{-\mathrm{n}_{\mathrm{s}-1}}\right)$

where $\operatorname{th}^{*}(s)$ is the optimum threshold in $s-$ th quad.

Theorem 4-1:

The scanning raster is begin at the topmost edge point and scanning from left to right, the horizontal interval $m$ can be counted by the pixel number. The optinun threshold in eq. 5-9 approximates to object edge in $Q_{5}$, if the pixel number is very small. Such that,

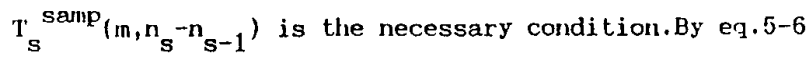
$\left|e_{s}\right|=\left|Q_{s}-Q_{s}^{\text {samp }}\right|$

$\left.=\mid R_{s}\left(m, n_{s-1}\right)+T\left(m, n_{s}-n_{s-1}\right)-R_{s} \operatorname{semp}_{\left(m, n_{s}-1\right.}\right)-T_{s}$ sanlp
$\left(m, n_{s}-n_{s-1}\right) \mid$ and the optimum threshold in the $\mathbf{s}^{-t h}$ Quad is defined as $\mathrm{IH}^{*}(\mathrm{~s})$ where 
$\mathrm{Th}^{*}(\mathrm{~s})=\mathrm{T}_{\mathrm{s}}^{\text {samp }}\left(\mathrm{m}, \mathrm{n}_{\mathrm{s}}-\mathrm{n}_{\mathrm{s}-1}\right)$

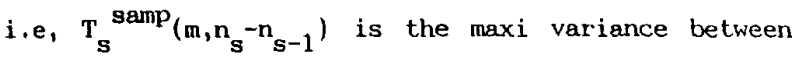
two similar edges. If there are more than two quads in the Quad String Array then the minimum optimum threshold string $\mathrm{Th}^{*}(\mathrm{~s})$ can be used as standard threshold.

$\mathrm{TH}^{*}(\mathrm{~s})=\underset{\mathrm{s}=1}{\#} \mathrm{Th}^{*} \mathrm{~s}=\underset{\mathrm{s}=1}{\#} \mathrm{~T}_{\mathrm{s}}^{\operatorname{samp}}\left(\mathrm{m}, \mathrm{n}_{\mathrm{s}}-\mathrm{n}_{\mathrm{s}-1}\right)$

$=\left[\mathrm{T}_{1}{ }^{\text {samp }}\left(\mathrm{m}, \mathrm{n}_{1}-\mathrm{n}_{0}\right), \mathrm{T}_{2}{ }^{\text {sarnp }}\left(\mathrm{m}, \mathrm{n}_{2}-\mathrm{n}_{1}\right), \ldots \mathrm{T}_{\mathrm{s}}^{\text {samp }}\left(\mathrm{m}, \mathrm{n}_{\mathrm{s}} \mathrm{n}_{\mathrm{s}-1}\right)\right]$

$(4-11)$

where "\#" is the string connection as defined in eq. (4-7), in eq.(4-11), it is the representation of optimun threshold string. There is an identification mapping $\epsilon$, which may not be defined on the all ranges of $\Sigma$, then

$\epsilon:\left\{e_{s}\left\langle T_{s}^{\text {samp }}\left(m, n_{s}-n_{s-1}\right)\right\} \ldots-\ldots\right.$

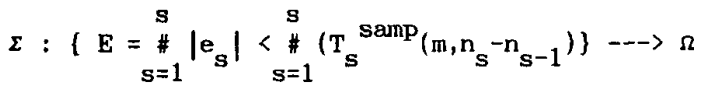

where $\Omega=\left\{w_{1}, w_{2}, \ldots w_{S}, \ldots w_{S}\right\} ; " \rightarrow-$ is a mapping relation.

Lemma 4-2:

By the definition of $Q_{s}$, the vertical pixel number for

standard sample image and unknown image are $\mathrm{N}_{\mathrm{s}}^{\text {samp }}$ and $\mathrm{N}_{\mathrm{s}}$ respectively.

$$
\text { If } \quad\left|\mathrm{N}_{\mathrm{s}}-\mathrm{N}_{\mathrm{s}}^{\text {samp }}\right|<=2 \mathrm{~T}_{\mathrm{s}} / \mathrm{m}
$$

m:horizontal pixel number

then $\quad: Q_{s} \rightarrow-\rightarrow W_{s}$ where $W_{s} \in \Omega$

Proof:

$\tilde{m}, \tilde{n}$ are very small and finite; $T_{s}=1 / 2 \tilde{m}, \tilde{n} ; \tilde{n}=2 T_{s} / m$ and $\tilde{n}=n_{s}-n_{s-1}$.

Corollary 4-1:

If the length of horizontal and vertical string are $\mathrm{m}, \mathrm{n}$ respectively which are positive real number, then the slope of image edge is determined by the following equation.

$$
\begin{aligned}
\text { slope }<=|1| \text { as } \tilde{m}>=\tilde{n} \\
\text { slope }<=|1| \text { as } \tilde{m}<=\tilde{n}
\end{aligned}
$$

Lemma 4-3:

By the definition of $Q_{s}, \tilde{m}=\tilde{n}, \tilde{m}, \tilde{n}$ are positive real number. If $m$ is constant for both input and sample pattern and

$$
\begin{aligned}
& \mid n_{i}-n_{i} \text { samp } \mid \ll n_{s}-n_{s-1}, s=1,2, \ldots s, i=1,2, N, \\
& \text { Then } \varepsilon: Q_{s} \rightarrow W_{s} \text { where } W_{s} \in \Omega \\
& (4-17)
\end{aligned}
$$

Lemna 4-4: Markovian Matching Block function The horizontal coordinate of Markovian matching block function is $\left(\tilde{m}_{1}, \tilde{m}_{2}, \ldots \tilde{m}_{s}\right)$ which is the horizontal segementation sequence. It is not necessary for i"s equal to $\tilde{m}_{s-1}$ as the object image edge is convex \& concave scene. If the object image edge is monodecreasing, for simplicity, the horizontal segmentation can be $0 m_{1}=\dot{m}_{1} m_{2}=m_{2} m_{3} \ldots$ and $o_{1}=\tilde{m}_{1}, m_{1} m_{2}=$ $\tilde{m}_{2}, \ldots m_{s-1} m_{s}=\tilde{m}_{s}$. The vertical coordinate of matching function can be segmented in the same process and the sequence is $\left\{\quad \tilde{n}_{1}, \tilde{n}_{2}, \ldots \tilde{n}_{s}\right\}$. We have the following relation:

$$
1 / 2 \tilde{n_{s} \tilde{m}_{s}}=T_{S}^{\text {samp }}
$$

where $\mathrm{T}_{\mathbf{s}}^{\text {samp }}$ is the triangular area in $Q_{\mathbf{S}}^{\text {samp }}$, it may be finite fixed value. Such that we can rewrite eq. $4-19$ eq. $4-20$

$$
\tilde{n}_{s}=2 T_{s} \text { samp / } \tilde{m}_{s}
$$

and $T_{s}$ samp is the maxi variance in Quad $s$ for Markovian matching block,

In Fig.5, suppose the curve $\mathrm{C}$ is divided into three Quads $Q_{1}, Q_{2}$ and $Q_{3}$. By the eq. (3-4):

$$
\begin{aligned}
& \mathrm{Q}_{1}=\mathrm{R}_{1}+\mathrm{T}_{1}=0+\mathrm{T}_{1} \\
& \mathrm{Q}_{2}=\mathrm{R}_{2}+\mathrm{T}_{2} \\
& \mathrm{Q}_{3}=\mathrm{R}_{3}+\mathrm{T}_{3}
\end{aligned}
$$

The basic assumption is that "T samp" is the maxi variance for Quad $s$, that is, in Quad $Q_{1}, T_{1}$ is the maxi tolerance and $T_{2}$ is the maxi tolerance for $Q_{2}$ etc. The curve $C_{1}$ in Quad $Q_{1}$ and $Q_{2}$ is similar to $C$, iff $\left|\mathrm{C}_{1}-\mathrm{C}\right|<=\mathrm{TH}^{*}(\mathrm{~s})$.

By eq. $(4-10)$ and $(4-11)$ :

$\left|C_{1}-C\right|={ }_{i=1}^{2}\left|e_{i}\right|=\left[T_{s}^{\text {samp }}, A_{1}+T_{2}^{\text {samp }}-A_{2}\right](4-22)$ if $A_{1}=A_{2}$ then $\left|C_{1}-C\right|<=\left[\mathrm{T}_{1}{ }^{\text {samp }}, \mathrm{T}_{2}^{\text {samp }}\right]=\mathrm{TH}^{*}(\mathrm{~s})$ then we can say " $C_{1}$ is similar to $C$ ".

In the other case if the tolerance is negative, then by Fig. 6 :

$$
C_{2}-C=-\left[T_{1},\left(A_{1}^{\prime}+T_{2}^{\prime}-A_{2}^{\prime}\right)\right]
$$

where $\mathrm{T}_{1}=\mathrm{T}_{1}$ samp

$$
\begin{aligned}
& t_{2}=T_{2}^{\text {samp }} \\
& \text { and if } A_{1}^{\prime}=A_{2} \text { ' }
\end{aligned}
$$

$$
\text { then }\left|\mathrm{C}_{2}-\mathrm{C}\right|=\left[\mathrm{T}_{1} \text { sanp }, \mathrm{T}_{2}^{\text {samp }}\right\}=\mathrm{TH}^{*}(\mathrm{~s})
$$

that is, $\mathrm{C}_{2}$ is similar to $\mathrm{C}$. The above two cases are the extreme condition for similarity, and any curve Jays between these two extreme conditions can be recognized as similar curve for $\mathrm{C}$. The Markovian matching block function is sketched as following: Fig.7. $L$ is the histogram of $\tilde{n}_{s} \tilde{m}_{s}$ for standard curve $C$ $\mathrm{L}_{1}$ is the histogram of $\mathrm{n}_{\mathbf{s}} \mathrm{m}_{\mathbf{s}}$ for standard curve $\mathrm{C}_{1}$ $L_{2}$ is the histogram of $\tilde{n}_{s} \tilde{m}_{s}$ for standard curve $C_{2}$ Now, we process the above algorithms to Quad $Q_{2}$ and $Q_{3}$ 
, and suppose the tolerance in $Q_{1}$ will not influence the variance in $Q_{2}$ and $Q_{3}$ then we can sketch the Markovian matching block from $Q_{2}$ again. The Markovian matching block function is shown as Fig.8.

We combine Fig.7 and Fig.8 together then we get the following result and shown in fig.9.

Any curve lays inside the block area shown in Fig. 8 is similar to curve $C$. There is an another example shown in Fig.9, the curve in that example is divided into 6 Quads. If the histogram of $\tilde{n}_{s} \tilde{m}_{s}$ for any unknown curve is laid inside the Markovian matching block of $\mathrm{C}$, then we can say that the unknown curve is similar to the standard curve C.

$\Sigma$ : \{Histogram of $\tilde{n}_{s} m_{s}^{\sim}$ for unknown object lay inside the normalized Markovian matching block) $\rightarrow \Omega(4-24)$

By the equation $4-20, \tilde{n}_{s}$ is variable and the histogram of $\tilde{n}_{s} \tilde{m}_{s}$ segments is defined as histogram function which is the recognition threshold in String Quad Array. The optimum threshold $\operatorname{Th}^{*}(\mathrm{~s})$ is defined as the boundary distance of matching block in each stage $s$. If the histogram $\tilde{n}_{\mathbf{s}} \tilde{m}_{\mathbf{s}}$ of unknown object is laid inside the matching block of standard pattern, then these two objects are similar each other.

Corollary 4-2:Defined $W(T)$ as the domain of the Markovian matching block, and $"=\cong \Rightarrow "$ is the representation of "within the matching function threshold" then

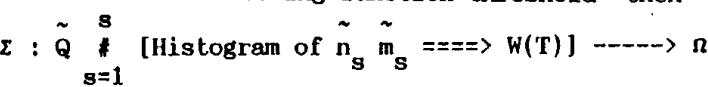

\section{RANDOM WALK APPROACH}

Random walk technique is further discussed by the view points of vertical pixels recursion string approach and vertical recursion Quad string sethod, By the ana1ysis of Pseudo Quad Potential function array which is shown in Fig.11.

$$
\tilde{Q}=\left\{Q_{1}, Q_{2}, \ldots Q_{s}, \ldots Q_{S}\right\}
$$

It is not necessary for $\tilde{m}_{s-1}=\tilde{m}_{s}$ and $\tilde{n}_{s-1}=\tilde{n}_{s}$.

In Fig.11, every Quad $Q_{s}$ is the combination of pixel cell array, every rectangular and triangular in $Q_{s}$ can be expressed by a pixel cell. By eq. 3-7, every odd Quad is convex and concave for every even Quad.

By the using of $Q_{s}$, there are two recognition approaches, one is vertical Quad string the other is vertical pixel string, the former has less recognition time but low recognition rate, the latter has high recognition rate.

Assumption 5-1:

As shown in Fig.11, Quad $Q_{S}$ is the combination of rec-

tangulars and triangulars. Every rectangular and triangular are expressed by a pixel set, but the rectangular area is far away from the object image. Such that, the shape of object image edge can be expressed by the triangular pixel cell approximately.

Definition $5-1$.

Usually, the shape of object edge is irregular flunctuating, the monodecreasing or monoincreasing can be defined by the equations $5-1$ and 5-2.
$\left(Q_{2 s-1} ; s=1,2, \ldots s / 2\right)-->$ convex scene
$\left(Q_{2 s} ; s=.1,2, \ldots s / 2\right)--\rightarrow$ convex scene

where convex scene is the representation of monodecreasing edge and convex scene is the representation of monoincreasing edge.

Definition 5-2:

$\Omega$ is defined as the image edge string of every Quad. then $\Omega=\left(w_{1}, w_{2}, \ldots . w_{s}, \ldots . w_{s}\right)$

(4-1)

The shape of edge mapping by $Q_{2 s-1}$ and $Q_{2 s}$ are different. ie,

$\left(Q_{2 s-1} ; s=1,2, \ldots s / 2\right)==^{*}=\Rightarrow\left(w_{2 \dot{s}-1} ; s=1,2, \ldots s / 2\right)$

$\left(Q_{2 s} ; s=1,2, \ldots s / 2\right)==^{*}==>\left(W_{2 s} ; s=1,2, \ldots s / 2\right)$

where $"={ }^{*}==>$ is the representation of edge "similarity"

Lemina 5-1:

By the equation $3-7$, edges mapping by $Q_{2 s-1}$ and $Q_{2 s}$ is monodescending and monoascending respectively. By the assumption 5-1, Quad is the combination of $R_{s}$ and $T T_{S}$ where $R$ is the representation of background and $T$ is the neighborhood representation of object edge. Due to the change of monodecending and monoascending, the sign variance of $T$ is defined by equation $5-5$ and 5-6.

$$
\begin{aligned}
& \left(\mathrm{T}_{2 \mathrm{~s}-1} ; \mathbf{s}=1,2, \ldots \mathrm{s} / 2\right) \ldots-^{*}-\rightarrow+1 \\
& \left(\mathrm{~T}_{2 \mathrm{~s}} ; \mathrm{s}=1,2, \ldots \mathrm{s} / 2\right) \ldots{ }^{*}-\rightarrow-1
\end{aligned}
$$

where " + " is the vertical downward movement of one ce11 and "-" is the vertical upward movement of one cell That is, what $R_{s}$ is known, then the progress of $T_{s}$ is a kind of Vertical Binary Random Walk.

where "--*-->" is the direction of random walk. Lenma 5-2: For every $Q_{2 s-1}$ and $Q_{2 s}$ which are $f$ it for the matching block function threshold defined in Lenmu 4-4 can be classified into feasible region. The decision mapping $E$ is defined by eq. $5-7$ and $5-8$ corresponding to eq. (5-5) and (5-6) respectively.

$$
\begin{aligned}
& {\left[Q_{2 s-1} ; s=1,2, \ldots s / 2=\Rightarrow W(T)\right] \rightarrow \Omega_{2 s-1}} \\
& {\left[Q_{2 s} ; s=1,2, \ldots s / 2=\Rightarrow W(T)\right] \rightarrow \Omega_{2 s}}
\end{aligned}
$$

where $"=\Rightarrow W(T) "$ is the representation of "within the matching function threshold", as defined in corollary 5-2 and it can be proved by eq, $6-3$ and $6-4$. Lemma 5-3: Quad $Q_{\mathrm{s}}$ referred is shown in Fig. 12.

The pixel cell of $Q_{s}$ can be divided by eq. 6-9 and 6-10

$$
\begin{aligned}
& m_{s}=\left(m_{s 1}, \ldots m_{s r}{ }^{\prime}, \ldots m_{s R}{ }^{\prime}\right), \quad r=1,2, \ldots R \\
& n_{s}=\left(n_{s 1}, n_{s 2}, \ldots, n_{s r}{ }^{\prime}, \ldots, n_{s r}{ }^{\prime}\right), r=1,2, \ldots, R, \quad(5-10) \\
& \text { where } m_{s 1}=M_{s-1}, m_{s r}=M_{s}, n_{s r}=N_{s-1}, n_{s r}{ }^{\prime}=N_{s} \\
& \varepsilon:\left[Q_{s}\left(m_{S r}, n_{s r},\right) ; r=1,2, \ldots, R=\Rightarrow W(T)\right] \rightarrow W_{S}(5-11) \\
& \Sigma:\left[Q_{\mathrm{s}}\left(\mathrm{m}_{\mathrm{sr}}, \mathrm{n}_{\mathrm{sr}}\right) ; \mathrm{r}=1,2, \ldots, \mathrm{R}===\mathrm{W}(\mathrm{T})\right]-\ldots \mathrm{n} / \mathrm{l}(5-12) \\
& \text { where } r=1,2, \ldots R \text { and } r^{\prime}=1,2, \ldots, R^{\prime} \text { and } Q_{s}\left(m_{s r}, n_{s r}{ }^{\prime}\right) \\
& \text { is the pixel cell quad array of } Q_{S} \text {. } \\
& \text { Theorem 5-1: } \\
& \text { By the algorithm of Random walk, system recognition }
\end{aligned}
$$


can the implemented by the vertical binary random walk - If the unknown object fits for eq. 5-7, 5-8, 5-11 and5-12 then following equation is existed.

: $\left\{Q_{2 s-1} ; s=1, \ldots s / 2\right.$ (monodecending scene) $\left.=\Rightarrow W(T)\right]$

$\rightarrow \Omega_{2 \mathrm{~s}-1}$

: $\left[Q_{2 s} ; s=1,2, \ldots s / 2\right.$ (monoaccending scene) $\left.==>W(T)\right]$

$-\rightarrow R_{2 s}$

This can be proved by Lemma 5-2 and Lerma 5-3.

Theorem 5-2:

$P\left[\left(m_{s r}, n_{s r}\right) \mid\left(m_{s(r-1)}, n_{s}\left(r^{\prime}-1\right)\right)\right]$ is defined as the protuability which transferred form the pixel $\left[\mathrm{m}_{\mathrm{s}}(\mathrm{r}-1)\right.$ '

$\left.n_{s}\left(r^{\prime}-1\right)\right]$ to the pixel $\left[m_{s r}, n_{s r}\right.$ '].

$$
\begin{aligned}
& \text { If } P\left(m_{s r^{\prime} n_{s r}}\right)=1, \\
& \text { then } \Sigma:\left(Q_{2 s-1} \cup Q_{2 s}\right) \rightarrow \infty
\end{aligned}
$$

where $s=1,2 \ldots . s, r=1,2 \ldots R, r^{\prime}=1,2 \ldots R^{\prime}$. This can be proved by Lemma 5-3 and theorem 5-1.

\section{EXPERIMENT RESULTS}

As discussing in scention III and IV, there are three different types object used in this experiment, these objects are handtool, human face and aircraft. The Vertical String Array, Random Walk recognition algorithms are applied on these test objects respectively. The experiment results are shown as the following tables.

Table 1 :

Correct

Recognition

II.a

H.b

H.c

HI, d

Vertical String
Array approach
0.93
0.90
0.90
0.90

Random Walk
approach
0.95
0.93
0.93
0.96

Table 2:

Correct

Recognition

Face A

Vertical string

Array approach

0.62

0.55

Random Walk

approach

0.70

0.67

Table 3:

Correct

Recognition

$$
\text { F-16 }
$$

$M i g-25$

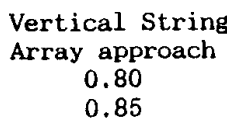

Random Walk

approach

0.90

0.89

\section{CONCLUSIONS}

1. The recognition algorithms discussed in this paper utilize the object background quad as the basic elements. the recognition process is beginning the topnost point of object edge, and it is especially useful in eral-time recognition.

2 . The object images are different as the view direclion changed. The aircraft is viewed at upward and face to face direction, the variance between two continuous image is also stored in the computer memory.

3. The matching of .principal axis direction between unknown object image and standard pattern is implemented by the Circular Layer Code techniques.

4. It is better to normalize the object image within the scarner device, this can be done by hardware or software such that memory size as well as the processing time of main processor can reduced to minimum.

5. The edge of input image will be steplized by the Siring Quad Array approach, the variance between step form edige and analog edge is the major factor for inisrecognition.

6. For the hollow object, the algorithm can apply on it and the object can be recognized within one fram's scarming.

7. It is not necessary to rotated the object image in Pseudo Quad plane, if we modified the associated software prograun.

8. The major error of recognition is due to the not matching of principle axis between standard pattern and unlunown object.

\section{REFERENCES}

[1] Y. L. Ma, "pattern recognition by Markovian dynamic programing," in Proc. 7th Int. Conf. Pattern Recognition, Montreal Canada, Jul. 30-Aug, 2, 1984, pp. 1259-1262.

[2] Y. L. Ma and J. S. Jour, "New probability model for Chinese character recognition," in Proc. 7th Int. Conf. Pattern Recognition, Montreal, Canada, Jul. 30-Aug. 2, 1984, pp. 370-373.

[3] Y. L. Ma, and J. S. Jour, "Pattern recognition by dynamic look-up -table programming," in Proc. IBOON, Tokyo, Japan, Oct. 22-26, 1984, pp. 1142-1147.

[4] Y. L. Ma, and S. Y. Jang, "pattern Recognition By Circular Layer Code approach," Prof.of 8th IAPR Paris, France, Oct. 1986, pp. 783-786.

[5] Y. L. Ma, chailo Ma and T. Y. Tu, "Moving Image Signal Processing by Markovian Random Walk approach," SPIE Conference of Pattern Recognition and Signal Processing. ORLANDO, U. S. A. April 7-8 1988.

[6] D. Nitizan, "Three dimensional Vision structure for Robot Application," IEFE Train on Pattern Anal and Machine Intel, vol. 10, No. 3, May 1988. pp. 291-309.

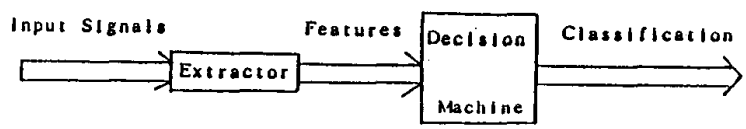

FIg. I Clossiflication block.

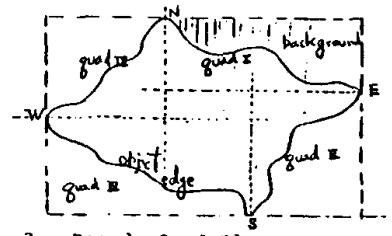

Fig. 2. Pseudo Ouad Plane.

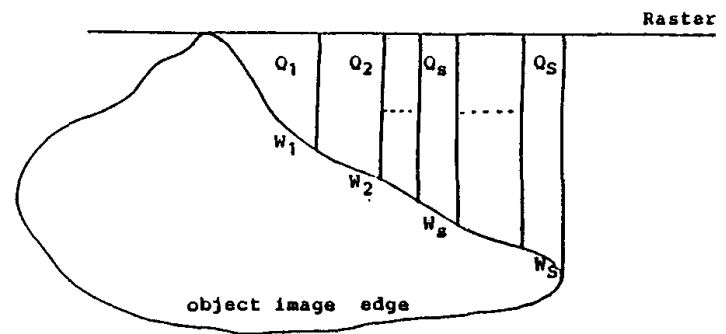

Fig. 3. monodecreasing quad plane.

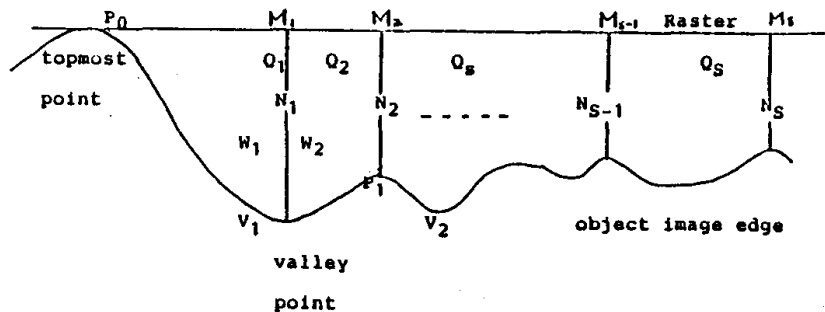

Fig. 1. moxodescending and momoascending 


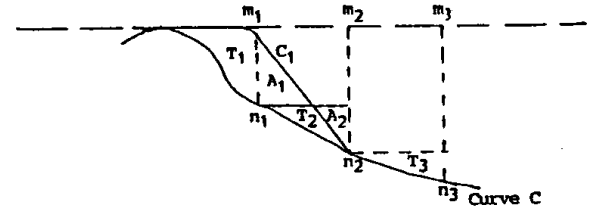

Fig. Positive varlance for two simflar curves

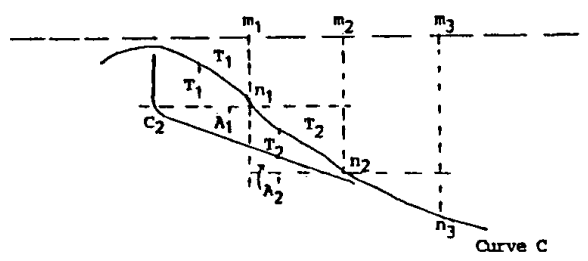

rig. Negative variance for two ofmilar curves

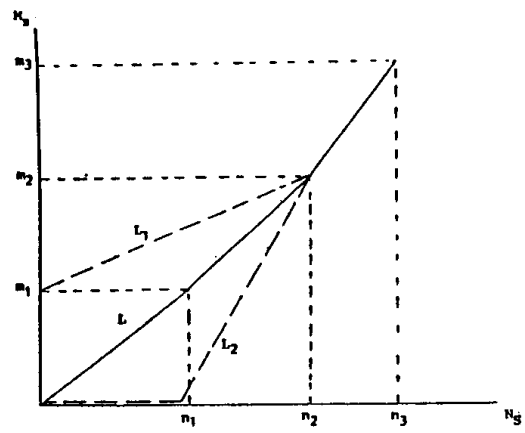

Fig.7 Markovion matching block function for two quads curve

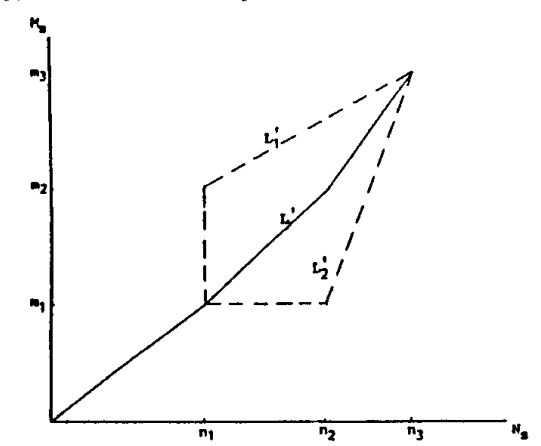

F1g.8 Markovian matching block function for secondary quad.

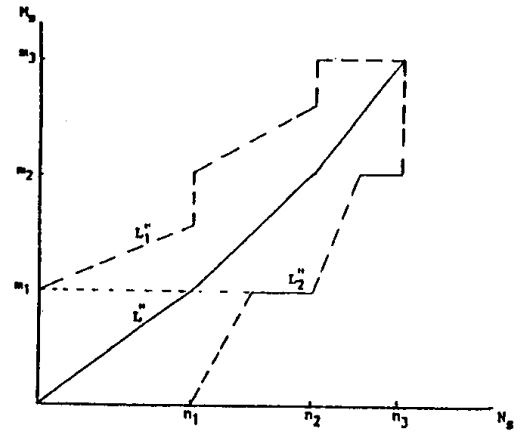

Flg.9 Markovian matching function for three guads curves

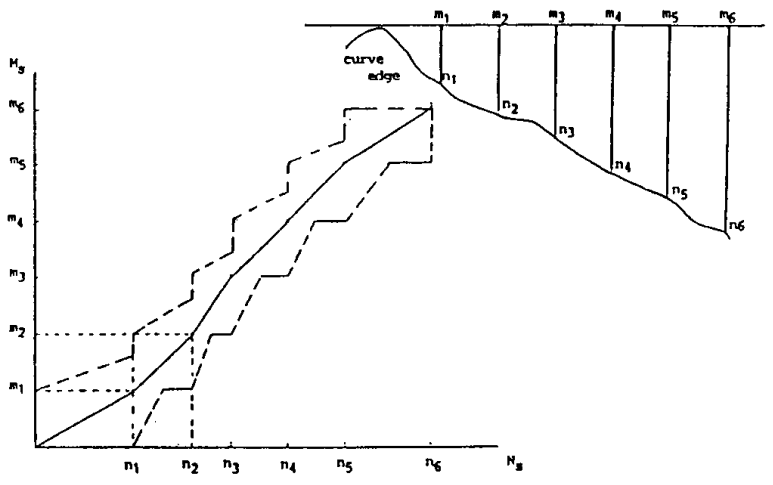

Fig.10 Markovian matching block function and 1 ts' relative curve

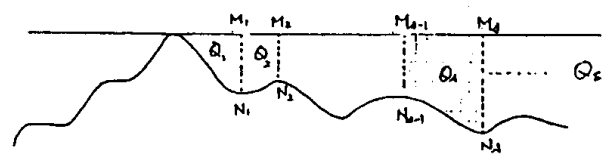

Fig. 11 Image of fluctuating edge.
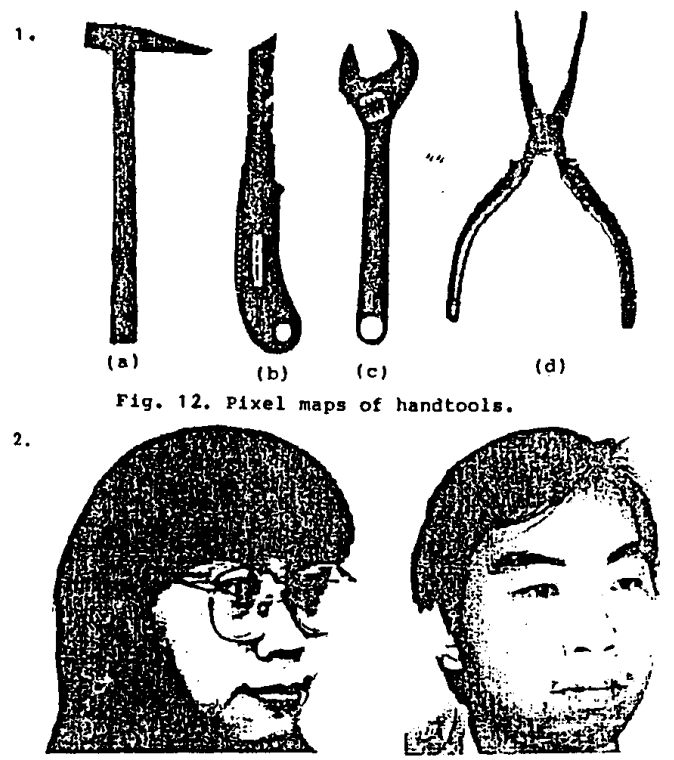

(a) FACE A

(b) FACE D

F19. 13. P1xel inaps of human faces.

3.

(a)

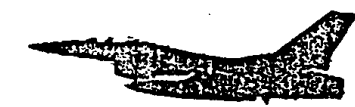

(b)

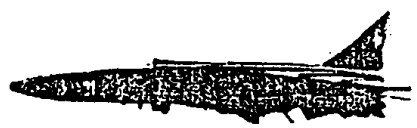

F1g. 14. P1xel maps of aircraft F-16 and Mig-25. 